\title{
Unpredictability of Seizures and the Burden of Epilepsy
}

Andreas Schulze-Bonhage, Anne Kühn

1.1

Introduction

About $0.8 \%$ of the world's population suffer from epilepsy. The classical definitions [1] of epilepsy deem the 'spontaneous occurrence' of recurrent seizures to be essential for a seizure disorder to be diagnosed as 'epilepsy'. Spontaneous seizures stand in contrast to situation-related seizures which are thought to be triggered by precipitants of the ictal event. The term 'spontaneous' reflects our present lack of understanding about the mechanisms underlying interictal-ictal transitions. Indeed, the relative contribution of genetic make-up in determining the individual 'seizure threshold', of intrinsic fluctuations in EEG dynamics, and of external factors, toward the development of an ictal state are unknown. It follows from this that it is currently not possible to predict the critical time points at which the interictal-ictal transition and the manifest 'seizure' take place.

Overt symptoms in epilepsy are virtually absent during the interictal period (as long as no elaborated diagnostic procedures are performed). This means that patients might be thought to suffer from the disease only during the brief paroxysmal episodes. A mean seizure frequency of three such events per month in the average adult pharmacoresistant patient [2] and an average seizure duration of 1-2 minutes [3-5] corresponds to a symptomatic period that effectively lasts less than one hour per year. Even if impairments during the postictal period are taken into consideration, most epilepsy patients are in a functional interictal state without obvious impairments for $95-99 \%$ of the time.

Despite the relatively low percentage of absolute time in the ictal state, the unpredictability of seizures still overshadows the life of most epilepsy patients. The simple fact of not knowing when an interictal-ictal transition may occur can greatly accentuate the subjective impact of potentially imminent seizures on the patient's everyday life [6-10]. Seizure unpredictability has major implications for patients, including its impact on medical diagnosis, its current role in determining the therapeutic approach, and its practical clinical consequences for the patients,

Seizure Prediction in Epilepsy. Edited by Björn Schelter, Jens Timmer and Andreas Schulze-Bonhage Copyright @ 2008 WILEY-VCH Verlag GmbH \& Co. KGaA, Weinheim ISBN: 978-3-527-40756-9 
21 Unpredictability of Seizures and the Burden of Epilepsy

ranging from the risk of complications secondary to epileptic seizures to socio-legal constraints and psychiatric comorbidity of the disease.

1.2

Medical Implications of Unpredictability

1.2.1

Diagnostic Uncertainty

Based on the intermittent character and brief duration of epileptic seizures, the patient will meet a physician in the vast majority of cases in the interictal state only, even if treated for years. This may delay or considerably reduce the chances of correct diagnosis. This is particularly true when additional features of epileptic attacks, such as partial or complete loss of consciousness during a seizure and retrograde amnesia for ictal events, render it difficult to obtain sufficient circumstantial information on the paroxysmal events, from the patient's history [11]. Documentation and analysis of paroxysmal events using video-EEGmonitoring is the gold standard for determining the diagnosis of epilepsy [12-14], but the unpredictability of the events may make this diagnostic method unfeasible in cases with low frequency.

\section{2 .2}

\section{Treatment Options}

Whereas in other paroxysmal diseases, such as migraine, an effective acute intervention is possible in the early phase of an attack, the brief duration of most epileptic seizures places a severe limitation on the potential effect of acute treatment. Medical treatment will not take effect if systemically applied before a seizure has spontaneously ended because of the pharmacokinetic delays between application and efficacy at their targets associated with drug absorption and distribution. Brain stimulation offers advantages in this respect. But at present, stimulation is only used, albeit widely, in the form of vagus nerve stimulation, which can be interactively triggered by the patient him or herself or another person by using a magnet, once the clinical symptoms become overt [15]. So far, the efficacy of ictal vagus nerve stimulation has yet to be studied in detail in the human; limited efficacy may be related to rapid spread of ictal activity, particularly if clinical seizure onset precedes its activation [16]. Rapid detection methods may provide new opportunities in a closed-loop setting [17].

The unpredictability of seizure occurrence generally results in the treatment being performed continuously over time in order to prevent interictal-ictal transitions. In treatments using brain stimulation via the vagus nerve or directly using intracranially implanted depth electrodes, more or less continuous stimulation patterns with variable duty cycles are applied $[18,19]$. The vast majority of patients 
have to take medication every day over a period of years, independent of actual seizure frequency. As the aim is to achieve a steady drug concentration at the target site, many drugs require an intake of at least twice a day, in some cases up to four times a day, to avoid breakthrough seizures [20], which may pose problems for patient compliance [21]. Drug level fluctuations in the case of irregular intake or of intercurrent alterations in resorption, metabolism or pharmacodynamic interactions may themselves trigger 'withdrawal' seizures [22-25].

The unpredictability of seizure occurrence is not the only burden for patients because of the regular intake requirement of antiepileptic medication. A continuously high level of antiepileptic medication is necessary for an optimal control of seizure frequency but is often accompanied by side effects. These typically encompass unspecific CNS-related effects like tiredness, dizziness, blurred vision or headaches, but may also include more specific impairments in cognition including difficulties with attention, concentration or language functions [26]. In turn, such dose-dependent side effects during continuous intake often place constraints on the maximal efficacy a drug can achieve; the need for continuous long-term administration thus affects both efficacy and tolerability of present-day pharmacotherapy. Another problem related with the continuous long-term treatment is the loss of efficacy in certain drugs (e.g., benzodiazepines) which are effective only in an acute setting but not for a protracted period, this being due to the development of tolerance [27]. At maximally tolerated dosages with continuous systemic drug administration, about one third of current epilepsy patients continue to have epileptic seizures and are regarded as 'pharmacoresistant' [28]. Seizure prediction would therefore open new avenues for drug treatment; for example, using short-acting drugs like lorazepam, or the transient application of high drug dosages for acute seizure abortion which cannot be used in the long term.

\section{2 .3}

\section{Physical Risks}

Patients with epilepsy frequently suffer from seizure-related injuries [29-31]. These result in part from the loss of control over the motor system during a seizure, as in the case of falls during atonic or tonic seizures. Often, a limited reactivity to external stimuli plays a major role in epilepsy-related injures. The loss of consciousness or delayed reactivity may therefore lead to an increased accident risk of patients exposed to demanding road traffic, particularly if there is no preceding warning symptom [29]. Similarly, the risk of accidental physical harm is increased in a spectrum of sport activities [32]. Even at home, the risk of sustaining seizure-related injuries is greatly increased during everyday activities; examples of these are increased frequencies of burns during cooking or showering [33,34], or an increased risk of drowning in the bathtub [35], which is a major cause of death in epilepsy patients. The absence of any warning signal preceding a seizure may in fact increase the risk of accidental physical harm considerably. 
Although most antiepileptic drugs are remarkably well tolerated over many years, there is a spectrum of substance-dependent risks associated with continuous longterm application. The relative importance of such side effects may be different depending on age. Cognitive side effects of phenobarbitone and pro-apoptotic actions of several drugs may be particularly important in the developing brain [36], the hormonal effects of valproate [37] may have their greatest impact in fertile females, and the induction of ostepenia by enzyme-inducing drugs [38,39] may play a particular role in increasing the risk in the elderly for the development of pathological fractures. Other side effects of chronic intake like the development of cerebellar atrophy and polyneuropathy with phenytoin [40,41], disturbances of hormonal metabolism [42], the induction of mood disorders by several drugs [43] and the development of visual field constrictions with vigabatrin [44] are consequences of long-term intake and pose problems at any age. The task of controlling the development of side effects related to long-term therapy imposes considerable costs on the healthcare system, even if these effects are as rare as certain idiosyncratic reactions, like liver failure or bone marrow aplasia.

\section{3}

\section{Psychosocial Consequences of Unpredictability}

The unpredictability of seizure occurrence has psychological and social consequences that are often closely interrelated. Seizures that reoccur frequently and unpredictably are accompanied by a patient's objective loss of control associated with the reduced ability to steer motor behavior and by the subjective loss of control associated with feeling overwhelmed by the effects of the disease. This experience is particular to epilepsy, the consequences of which are reflected in the psychological concept of 'locus of control' and in the development of psychiatric symptoms of anxiety and depression that have, in turn, social implications.

\section{3 .1}

\section{Loss of Control}

The concept of 'locus of control' reflects the cognitive style of attributing events and actions to either internal or external factors. Internal factors encompass the person's own behavior, abilities or characteristics, whereas external factors include chance or misfortune on the one hand and actions of other persons on the other. This psychological concept thus deals with an individual's tendency to perceive events as being controlled either by themselves or by external forces [45]. This idea was originally formulated by Julian B. Rotter and recognises the importance of the individual's perception of causality as attributable to both 
intrapersonal determinants and the social context of behavior. Individuals tend to categorise situations according to their perception of success or failure and, in particular, according to the reason for their outcome. Depending on the individual's perception of causality, an internal locus of control reflects the belief in a positive reinforcement by his or her own action; whereas an external locus of control is associated with the expectancy of reinforcement by chance or by uncontrollable factors - as would apply in the case of unpredictably occurring seizures.

The concept of locus of control is crucial for achievement motivation and actions and for emotional reactions to social events. It may therefore influence compliance, in terms of outpatient attendance at epilepsy clinics and regular intake of antiepileptic medication, and be a contributory factor particularly in the occurrence of depressed mood and anxiety. Coping strategies and the well-being of patients with chronic diseases are generally associated with perceived locus of control [46], though not only in patients with epilepsy [47,48]. Children with epilepsy are, already at this early age, more likely to attribute control of events to external factors than are chronically ill children with diabetes or healthy controls. Children with epilepsy show also a lower self-esteem and a higher level of anxiety compared with their peers $[49,50]$.

Clinical research uses condition-specific locus of control scales like the Multidimensional Health Locus of Control scales by Wallston and colleagues [51]. Health locus of control mirrors the patients beliefs regarding perceived control over the disease and determines health-related behavior. The Multidimensional Health Locus of Control (MHLC) scale comprises three subscales: internality (I-HLC), chance (C-HLC), doctors and powerful others (P-HLC) [52]. Studies examining the attitudes of patients with epilepsy revealed weak perception of internal and strong perception of external health locus of control [50,53,54]. This pattern of internality and externality may result in a less effective adaptation of these patients with epilepsy to their illness and a lower engagement in beneficial health behavior and active coping strategies [54].

Recent studies additionally addressed associations between locus of control and self-efficacy or self-confidence, showing that a patient's internal locus of control correlates with his or her self-efficacy. Both are described as mastery variables influencing the patient's quality of life [55] and as predictors for psychological distress [56]. Accordingly, happiness with life and self-confidence are particularly low in patients with high seizure frequency [57].

Even though most patients with epilepsy do not feel that they have control over their seizures, more than fifty percent of patients believe that they can identify seizure precipitants like stress and fatigue correctly, and many have developed strategies by which they try to prevent occurrence of seizures or to abort them $[58,59]$. Self-control of seizures would 'elevate' the individual to the position of being able to regulate events and this shift in control expectancy may likely have a positive psychosocial impact. 'High controllers' and 'low controllers' can thus be distinguished according to their belief in their ability to exert control over their seizures, and this, again, correlates with scores on 
61 Unpredictability of Seizures and the Burden of Epilepsy

health locus of control scales $[53,60]$. Importantly, the degree of self-perceived seizure control practically manifests itself in patients who seek low-risk-for-seizure situations, avoid high-risk-for-seizure situations, and make attempts at seizure prevention.

\subsection{2}

\section{Problems with Coping Strategies}

Strategies such as seeking out low-risk-for-seizure situations and avoiding highrisk situations are considered behavioral coping strategies. These correspond at a cognitive level with propositions like 'Try to maintain some control over the situation' or 'Hope things will get better' [61], and may lead to a search for information, contact with other patients and support groups, and to keeping a seizure diary [62]. Whereas coping strategies may activate patients' resources and contribute to psychosocial adjustment and health [46], avoidance behavior may have negative social implications. Feeling stigmatised, patients with epilepsy are often ashamed of having publicly 'displayed' unpredictable seizures, and they may therefore tend to avoid leaving home. This can result in social withdrawal, isolation, lack of positive social interaction and experience, and, finally, in a loss of self-efficacy and a decline in quality of life.

\subsection{3}

\section{Depression and Anxiety}

Depression and anxiety are the most prevalent psychiatric disorders in adults with epilepsy [63] and already appear in one-third of children suffering from epilepsy. Both depression and anxiety may result in suicidal ideation and behavior, even in childhood [64]. About thirty percent of adult patients with epilepsy report suicide attempts [65], and the suicide rate of epilepsy patients is at least three times higher than that of the general population $[66,67]$, particularly in women [68]. Loss of control is a major psychological factor leading to depression and anxiety $[54,59]$. Epilepsy patients have low internal control beliefs and medium beliefs in the role of chance. The patient's tendency to attribute power to others correlates with the degree of anxiety. Depressivity is related with a more external attributional style and with loss of internal control beliefs $[46,69,70]$. This corresponds to the learned helplessness model of depression by Seligman [70]. Herein, the symptoms of depression such as passiveness, cognitive deficits, and problems with self-esteem, are due to a lack of contingency between a person's behavior and its consequences [71]. Unpredictably occurring seizures, particularly if accompanied by loss of motor control or consciousness, are paradigmatic for such a helpless situation. Correspondingly, patients with pharmacoresistant temporal lobe epilepsy undergoing surgical therapy show, preoperatively, a significant relationship between self-reported depression and external locus of control [72]. Postoperatively, the early anticipation of seizure freedom may already improve mood. 
1.3 .4

Immobility and Vocational Restrictions

The unpredictable occurrence of seizures results in the imposition of driving restrictions on epilepsy patients, necessarily so because the patient represents a danger to him or herself and to all other traffic participants. Most patients with epilepsy are therefore wholly reliant on public transport or on their personal social environment. Immobility may lead to vocational problems due either to problems accessing the place of employment or to driving a car being a job requirement [73].

Vocational restrictions may also be encountered by patients as a result of many indirect consequences of seizure unpredictability. The occurrence of seizures may be stigmatising in itself, but cognitive impairments related with the disease and side effects of antiepileptic medication often also place limitations on a patient's level of work performance and achievement. More than one-third of patients with active epilepsy are unemployed, while this applies to about ten percent of patients in remission. This serves only to heighten the dependency on the social security system [57] and to reinforce the feeling of subjective handicap. The range of vocational possibilities depends on seizure severity: the unpredictable loss of consciousness, falls, inadequate behavior and loss of motor control are particularly unfavorable [74].

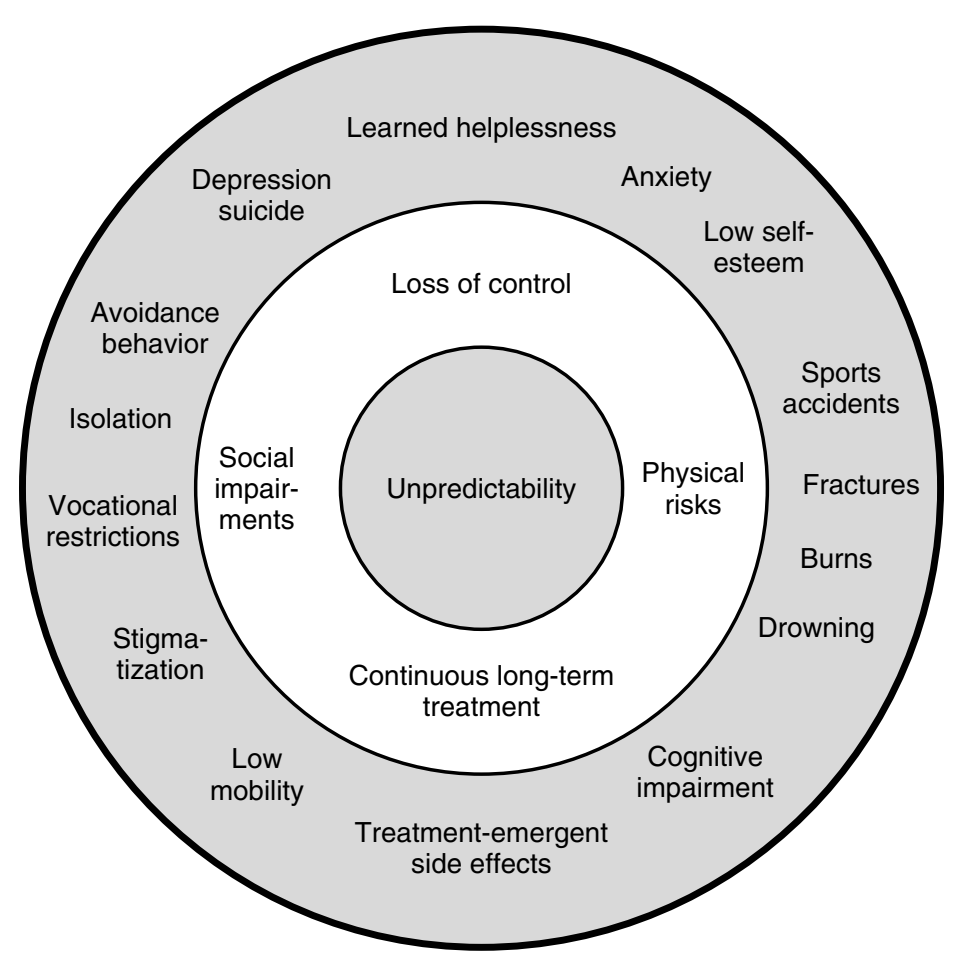

Fig. 1.1 Consequences of seizure unpredictability in epilepsy patients. 


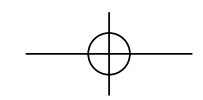

\begin{tabular}{l|l}
8 & 1 Unpredictability of Seizures and the Burden of Epilepsy
\end{tabular}

1.4

Conclusion

The development of techniques designed to predict epileptic seizure occurrence could make a considerable contribution to improving the well-being of patients in all areas discussed here and summarised in Figure 1.1. Progress in this field could facilitate medical diagnosis, and new timely drug delivery or stimulation techniques could be specifically targeted to intervene in the preictal brain dynamics that lead to a seizure. Intermittent therapy could offer major advantages both in efficacy and in long-term tolerability. Finally, reliable warning systems would reduce patients' risk of physical harm, might offer new windows of occupational opportunity and leisure activities, and could contribute to a change in the patient's perception of external locus of control, the feeling of helplessness and to secondary psychiatric problems.
\end{abstract}

\title{
References
}

1 Epilepsia 22, 489 (1981).

2 J. Bauer and W. Burr, Seizure 10, 239 (2001).

3 S. Jenssen, E. J. Gracely and M. R. Sperling, Epilepsia 47, 1499 (2006).

4 W. H. Theodore, R. J. Porter, P. Albert, K. Kelley, E. Bromfield,

O. Devinsky and S. Sato, Neurology 44, 1403 (1994).

5 O. Devinsky, K. Kelley, R. J. Porter and W. H. Theodore, Neurology 38, 1347 (1988).

6 F. Gilliam, R. Kuzniecky, E. Faught, L. Black, G. Carpenter and

R. Schrodt, Epilepsia 38, 233 (1997).

7 M. Hayden, C. Penna and N. Buchanan, Seizure 1, 191 (1992).

8 R. Ortiz and J. Liporace, Epilepsy Behavior 6, 620 (2005).

9 I. M. Elliott, L. Lach and M. L. Smith, Epilepsy Behavior 7, 664 (2005).

10 A. Jacoby, Soc. Sci. Med. 34, 657 (1992).

11 Rev. Neurol. (Paris) 156, 481 (2000).

12 S. Kipervasser and M. Y. Neufeld, Acta Neurol. Scand. 116, 221 (2007).

13 B. J. Steinhoff and A. SchulzeBonhage, EEG (Thieme, Stuttgart, 2006), chap. Langzeit-EEG, pp. 79-83.
14 M. Manford, J. Neurol. Neurosurg. Psychiatry 70 Suppl 2, II3 (2001).

15 G. L. Morris, Epilepsy Behavior 4, 740 (2003).

16 K. Götz-Trabert, C. Hauck, K. Wagner, S. Fauser and A. SchulzeBonhage, Epilepsia in press (2008).

17 I. Osorio, M. G. Frei, S. Sunderam, J. Giftakis, N. C. Bhavaraju, S. F. Schaffner and S. B. Wilkinson, Ann. Neurol. 57, 258 (2005).

18 C. Heck, S. L. Helmers and C. M. DeGiorgio, Neurology 59, S31 (2002).

19 W. H. Theodore and R. S. Fisher, Lancet Neurol. 3, 111 (2004).

20 M. Bialer, CNS Drugs 21, 765 (2007).

21 J. A. Cramer, R. H. Mattson, M. L. Prevey, R. D. Scheyer and V. L. Ouellette, JAMA 261, 3273 (1989).

22 J. C. DeToledo, R. E. Ramsay, M. R. Lowe, M. Greiner and E. A. Garofalo, Ther. Drug Monit. 22, 753 (2000).

23 H. Martínez-Cano, A. Vela-Bueno, M. de Iceta, R. Pomalima and I. Martínez-Gras, Pharmacopsychiatry 28, 257 (1995).

24 J. S. Duncan, S. D. Shorvon and M. R. Trimble, Epilepsia 31, 324 (1990). 
25 F. Buchthal, O. Svensmark and H. Simonsen, Arch. Neurol. 19, 567 (1968).

26 B. Gomer, K. Wagner, L. Frings, J. Saar, A. Carius, M. Härle, B. J. Steinhoff and A. SchulzeBonhage, Epilepsy Behavior 10, 486 (2007).

27 W. Löscher and D. Schmidt, Epilepsia 47, 1253 (2006)

28 P. Kwan and M. J. Brodie, N Engl. J. Med. 342, 314 (2000).

29 E. C. Wirrell, Epilepsia 47 Suppl. 1, 79 (2006)

30 P. Vestergaard, S. Tigaran, L. Rejnmark, C. Tigaran, M. Dam and L. Mosekilde, Acta. Neurol. Scand. 99, 269 (1999).

31 H. B. I. Persson, K. A. Alberts, B. Y. Farahmand and T. Tomson, Epilepsia 43, 768 (2002).

32 G. M. Howard, M. Radloff and T. L. Sevier, Curr. Sports Med. Rep. 3, 15 (2004).

33 I. C. Josty, V. Narayanan and W. A. Dickson, Epilepsia 41, 453 (2000).

34 M. C. Spitz, J. A. Towbin, D. Shantz and L. E. Adler, Epilepsia 35, 764 (1994).

35 C. A. Ryan and G. Dowling, CMAJ 148, 781 (1993).

36 P. Bittigau, M. Sifringer, K. Genz, E. Reith, D. Pospischil, S. Govindarajalu, M. Dzietko, S. Pesditschek, I. Mai, K. Dikranian et al., Proc. Natl. Acad. Sci. USA 99, 15089 (2002).

37 A. Verrotti, R. Greco, G. Latini and F. Chiarelli, J Pediatr. Endocrinol. Metab. 18, 423 (2005).

38 A. M. Pack and M. J. Morrell, Epilepsy Behavior 5 Suppl. 2, S24 (2004).

39 R. D. Sheth, B. E. Gidal and B. P. Hermann, Epilepsy Behavior 9, 601 (2006).

40 F. A. D. Marcos, E. Ghizoni, E. Kobayashi, L. M. Li and F. Cendes, Seizure 12, 312 (2003).

41 A. Bono, E. Beghi, G. Bogliun, G. Cavaletti, N. Curtó, L. Marzorati and L. Frattola, Epilepsia 34, 323 (1993).

\section{References $\mid 9$}

42 J. I. T. Isojärvi, E. Taubøll and A. G. Herzog, CNS Drugs 19, 207 (2005).

43 B. Schmitz, Epilepsia 47 Suppl. 2, 28 (2006).

44 L. V. Wilton, M. D. Stephens and R. D. Mann, BMJ 319, 1165 (1999).

45 J. B. Rotter, Psychol. Monogr. 80, 1 (1966).

46 K. Krakow, K. Bühler and H. Haltenhof, Seizure 8, 111 (1999).

47 M. C. Chung, E. Preveza, K. Papandreou and N. Prevezas, J. Affect. Disord. 93, 229 (2006).

48 M. C. Chung, E. Preveza, K. Papandreou and N. Prevezas, Psychiatry Res. 152, 253 (2007).

49 S. Correa, Psychol. Rep. 60, 9 (1987).

50 W. S. Matthews, G. Barabas and M. Ferrari, Epilepsia 23, 671 (1982).

51 K. A. Wallston, B. S. Wallston and R. DeVellis, Health Educ. Monogr. 6, 160 (1978).

52 K. A. Wallston, M. J. Stein and C. A. Smith, J. Pers. Assess 63, 534 (1994).

53 S. Spector, C. Cull and L. H. Goldstein, Epilepsia 42, 556 (2001).

54 A. A. Asadi-Pooya, C. A. Schilling, D. Glosser, J. I. Tracy and M. R. Sperling, Epilepsy Behavior 11, 347 (2007).

55 M. Amir, I. Roziner, A. Knoll and M. Y. Neufeld, Epilepsia 40, 216 (1999).

56 A. M. S. Wu, C. S. K. Tang and T. C. Y. Kwok, Aging. Ment. Health 8, 21 (2004).

57 M. F. O’Donoghue, D. M. Goodridge, K. Redhead, J. W. Sander and J. S. Duncan, Br. J. Gen. Pract. 49, 211 (1999).

58 C. A. Cull, M. Fowler and S. W. Brown, Seizure 5, 131 (1996).

59 M. R. Sperling, C. A. Schilling, D. Glosser, J. I. Tracy and A. A. Asadi-Pooya, Seizure (2007).

60 M. Rosenbaum and N. Palmon, J. Consult. Clin. Psychol. 52, 244 (1984).

61 M. Snyder, Int. Disabil. Stud. 12, 100 (1990).

62 J. Murray, Seizure 2, 167 (1993).

63 M. F. Mendez, R. C. Doss, J. L. Taylor and P. Salguero, J. Nerv. Ment. Dis. 181, 444 (1993). 


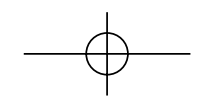

10 1 Unpredictability of Seizures and the Burden of Epilepsy

64 R. Caplan, P. Siddarth, S. Gurbani, R. Hanson, R. Sankar and W. D. Shields, Epilepsia 46, 720 (2005).

65 M. F. Mendez, J. L. Cummings and D. F. Benson, Arch. Neurol. 43, 766 (1986).

66 L. Nilsson, T. Tomson, B. Y. Farahmand, V. Diwan and P. G. Persson, Epilepsia 38, 1062 (1997).

67 J. E. Jones, B. P. Hermann, J. J. Barry, F. G. Gilliam, A. M. Kanner and K. J. Meador, Epilepsy Behavior 4 Suppl. 3, S31 (2003).

68 V. V. Kalinin and D. A. Polyanskiy, Epilepsy Behavior 6, 424 (2005).
69 B. P. Hermann, M. R. Trenerry and R. C. Colligan, Epilepsia 37, 680 (1996).

70 D. W. Dunn, J. K. Austin and G. A. Huster, J. Am. Acad. Child Adolesc. Psychiatry 38, 1132 (1999).

71 M. E. P. Seligman, Learned Helplessness (Freeman, San Francisco, 1975).

72 B. P. Hermann and A. R. Wyler, Epilepsia 30, 332 (1989).

73 H. R. Stöckli, Ther. Umsch. 64, 429 (2007).

74 P. Bülau and A. zur Verbesserung der Eingliederungschancen von Personen mit Epilepsie, Rehabilitation (Stuttg.) 40, 97 (2001). 